\title{
Near-IR Mode-Locked Laser Assisted Sintering and Morphological Engineering of Biomaterials - A New Approach for Integrative Manufacturing of Hard-Soft Tissues for in-Theatre Use!
}

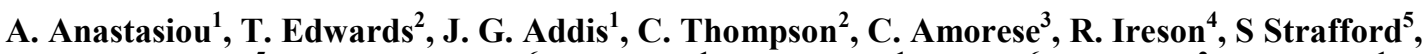

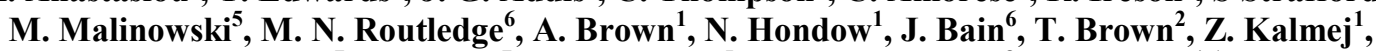 \\ M. Petruzzi ${ }^{7}$, R. Grassi ${ }^{7}$, M. S. Duggal ${ }^{5}$, P. V. Giannoudis ${ }^{8}$, and A. Jha ${ }^{*}, 1$ \\ ${ }^{1}$ School of Chemical \& Process Engineering, University of Leeds, Leeds LS2 9JT, UK \\ ${ }^{2}$ School of Physics \& Astronomy, University of St Andrews, North Haugh, St Andrews KY16 9SS, UK \\ ${ }^{3}$ I.C.M.E.A. SRL, Via Mongelli 11 - 70033 Corato (Bari), Italy \\ ${ }^{4}$ Glass Technology Services, 9 Churchill Way, Chapeltown, Sheffield S35 2PY, UK \\ ${ }^{5}$ School of Dentistry, University of Leeds, Leeds LS2 9JT, UK \\ ${ }^{6}$ M Squared Lasers, Venture Building, 1 Kelvin Campus, West of Scotland Science Park, Glasgow G20 0SP, UK \\ ${ }^{7}$ Dipartimento Interdisciplinare di Medicina, Università degli Studi di Bari Aldo Moro, Bari, Italy \\ ${ }^{8}$ Leeds Institute of Rheumatic and Musculoskeletal Medicine, University of Leeds, Leeds LS2 9JT, UK
}

\begin{abstract}
The emergence of mode-locked near-IR (NIR) lasers has opened novel and exciting opportunities in dental and orthopaedic medicine. In a mode-locked laser cavity the pulse duration and repetition rates may be controlled between 10-100s of femtosecond (fs) and kHz-GHz ranges, respectively. This unique capability for controlling the incident laser power in a near-IR mode-locked laser has been explored for studying the materials phase transformation, sintering and bonding mechanisms in calcium phosphate and chitosan/calcium phosphate suspensions as biomaterials. The investigation primarily focusses on interaction of such a laser in a linear regime, resulting in a plethora of phase combinations and morphologically controlled structures, which are well suited for in-theatre processing of hard-soft tissues for personalized therapy. In this article, the engineered medical device which combines the materials and laser power delivery at the point of tissue restoration is also discussed. The article exemplifies the case for enamel restoration using such a medical device, which then sets the scene for much wider use in tissue engineering.
\end{abstract}

\section{INTRODUCTION}

In mammalian bone and dental enamel, the naturally occurring hydroxyapatite (HAp formula $\left.\mathrm{Ca}_{5}\left(\mathrm{PO}_{4}\right)_{3} \mathrm{OH}\right)$ is the main bio-mineral. The crystalline form of HAp has an anisotropic structure with P63/m space group, with hexagonal symmetry which then attributes to the needle like shape in natural minerals. In such needle structure the c-axis is parallel to the needle axis. The lattice parameters of HAp with formula unit, $\mathrm{Ca}_{10}(\mathrm{PO} 4)_{6}(\mathrm{OH})_{2}$, are $a=9.423 \AA$ and $c=6.875 \AA[1]$. Although at the cellular level, the formation of bio-mineral starts with an amorphous state close to a neutral $\mathrm{pH}$ environment, however the local electrochemically active surface of collagens, which is the most abundant proteins in the bone, determines the thermodynamically stable physical state of the phosphate mineral by forming HAp in platelet forms along the a-axis of the hexagon lattice of HAp. The platelets then grows along the c-axis, supported by the anisotropic assemblies of collagen fibres [1]; the mechanism of which is controlled by osteoblast/osteoclast cells, the growth factor and the bone-morphogenic proteins. In the human body, the differentiation pathways for specific types of stem cells control the growth and physiological development of tissue types, which may be triggered by a specific growth factor for that tissue type! The apparent stem cell based differentiation mechanism in an osteogenic and angiogenic biomaterials provides a right environment for bone and blood vessel growth, respectively. By comparison the dental enamel and dentine tissue grow with the help of dental pulp stem cells (commonly found in $10-20$ millions in wisdom tooth), which may be expressed to become odontogenic, osteogenic and angiogenic with the help of a specific growth factor. Depending on the genetic lineages therefore the crystalline structure of HAp evolves to support specific hard-tissue functions; e.g. as in load-bearing bone, or as dentine or enamel. Once the stem cell expression reaches the end of genetic lineage, no further differentiation pathway can be expressed and this where the tissue structure regeneration stops. For this reason, unlike a damaged bone, the enamel tissue, once lost, never grows back again due to the lack of regenerative capacity [2].

With this background on regenerative capacities of bone and other types of harder tissues in human body, and the lack of regeneration of enamel, the article focus on the interaction of ultrafast lasers with bio-minerals for tissue regeneration which can be adopted for in-theatre use. Using the ultra-fast mode-locked lasers, the resulting material may provide the desired physiologically acceptable functional structures by controlling the average power and repetition rate which, otherwise, is impossible to achieve in a slow-pulsed near-IR lasers (e.g. Nd- 
YAG, or Ti-Sapphire). With the emergence and growing popularity of mode-locked lasers, the main objectives of our research in this area of biomaterial-mode-locked laser interaction is to develop tissue engineering technology and manufacturing capabilities for synthetic tissues for replacing the damaged tissues in-theatre by combining with the regenerative therapies already known and practiced in theatre during surgery. In this article, we cite examples of materials morphological structures using mode-locked near-IR lasers, developed for hard tissue engineering. An essential part of the mode-locked laser/bio-mineral interaction is also to work in a resonant regime in which the incident laser energy $\left(I_{o}\right)$ absorbed follows the Lambert-Beer's law: $I=I_{o} \cdot \exp (-\alpha \cdot \ell)$. Here $I$ is the scattered or transmitted intensity of photons in the medium, having a linear absorption coefficient, $\alpha$ and $\ell$ is the path length of the photons [3]. The resonant absorption of energy induces rapid phase transformation in the mineral and helps in forming bonds and changes the density of materials. This type of density change then may be used for controlling the average bulk density, by controlling the porosity, which are exemplified herein.

\section{METHODOLOGIES AND RESULTS}

The methods for synthesizing the calcium phosphate minerals; e.g. hydroxyapatite (HAp), brushite $\left(\mathrm{CaHPO}_{4} \cdot 2 \mathrm{H}_{2} \mathrm{O}\right)$ and fluorine-doped apatite are described in our previous articles [3-5]. For the analysis of resonant absorption of mode-locked femtosecond ( $\mathrm{fs}$ ) pulsed (120 fs) laser radiation at $1040 \mathrm{~nm}(1 \mathrm{GHz}$ repletion rates) using $\mathrm{Yb}^{3+}$-potassium tungstate crystal, we studied the phase transformation reactions in both the iron oxide $\left(\mathrm{Fe}^{3+}\right)$-doped and undoped brushite and apatite minerals. The doping with iron oxide $\left(\mathrm{Fe}^{3+}\right)$ was particularly chosen for enhancing the linear absorption of photons. In a previous article we also used the 120 fs-pulsed $1520 \mathrm{~nm}\left(\mathrm{Cr}^{4+}\right.$-tungstate) crystals [4] for $\mathrm{Er}^{3+}$-doped brushite. Also we had compared the influence of $1 \mathrm{GHz}$ repetition rate $\mathrm{Yb}^{3+}$-lasers on the resulting microstructures of doped and undoped calcium phosphate minerals $[3,5]$.

For demonstrating the restoration of damaged enamel using $\mathrm{Fe}^{3+}$-doped brushite and apatite and fs-pulsed laser radiation, we used bovine incisors in which the controlled acid-eroded lesions were created ex vivo, using citric acid medium maintained at $\mathrm{pH}=3.5$. The acid eroded lesion was typically $1.0-1.5 \mathrm{~mm}$ wide, $\sim 1 \mathrm{~mm}$ deep and $10 \mathrm{~mm}$ in length. We planned such a large lesion for the characterization of synthetic minerals before and after laser irradiation using the X-ray powder diffraction, Raman and FTIR spectroscopy, and profilometry. Also designing a large lesion for enamel restoration helps in the characterisation of bonded synthetically materials on bovine enamel surface using brushing trials and in-depth stress and image analyses, for ascertaining the efficacy of restorative procedure. In parallel with the bovine enamel related studies, we also investigated the bonding and adhesion mechanism using the die-pressed pellets of bio-minerals which were sintered in air muffle furnace above the phase transformation temperatures $\left(400^{\circ} \mathrm{C}-850^{\circ} \mathrm{C}\right)$. Using the die-pressed pellets we also compared the laser-radiation induced phased transformation in bio-minerals, for comparing with the transformed materials on and around the acid-eroded bovine enamel lesion and the air-muffle furnace heat treated materials. The results of phase transformation studies were also used for the analysis and estimation of potential temperature rise and energy accumulation in synthetic bio-minerals during tissue restoration process [3]. The effects of laser-induced cell and genotoxicity studies were also investigated in detail and these are reported elsewhere [5].

For materials delivery at the point of tissue restoration and its subsequent selective laser sintering [3] for bonding with the surrounding natural bovine enamels, a medical device was also designed using a CAD package, the schematic of the materials and laser delivery system is shown in Figure 1. The fibre-delivery system connected laser and materials delivery ports are identified in this figure. The middle and rear compartments of the medical device house the materials cartridge, piezo-electric assisted drive and power control systems, respectively. For assisting the ease of materials flow like tooth-paste through the delivery nozzle at the front of the device, the rheological properties of the bio-mineral suspension in water and ethanol was studied and for a stable flow of suspension, it was found that the suspension viscosity of more than 50 Pa-s must be maintained (see Fig. 2) to achieve the control delivery of sub-millilitre (ml) per minute.

An example of laser-irradiation experiments on the layers of brushite or apatite laid over enamel is shown in Fig. 3, in which the large regions of $\mathrm{Fe}^{3+}$-doped brushite or apatite were irradiated using a $1 \mathrm{GHz}$ repetition rate fs-pulsed mode-locked laser, operating at $1048 \mathrm{~nm}$. The laser source was fixed and the acid-eroded enamel with a defined lesion, into which the synthetic mineral was deposited, was translated under beam so that a laser beam spot of $30 \mu \mathrm{m}$ diameter can be pre-programmed to move along a track, as shown in Fig. 3. During translation along the mineral surface each $30 \mu \mathrm{m}$-spot irradiated the mineral surface for 0.3 seconds for initiating bonding and sintering with surrounding natural enamel. The extent of sintering was analyzed, as explained above using the phase analysis and scanning electron microscopic techniques. In Fig. 4 a typical coated surface of enamel is shown which was irradiated with a $1048 \mathrm{~nm}$ fs-pulsed laser with an average power of $420 \mathrm{~mW}$. The post irradiation microstructures, analyzed by SEM, are compared in Fig. 5, from which we clearly see the extensive evidence for melting, coalescence and above all intergranular and particle bonding with the surrounding phase. 


\section{CONCLUSIONS}

Based on the microstructural (in Fig. 5b) and phase analysis using X-ray diffraction (not shown here) [3-5], it is evident that the phases identified are consistent with the thermal analysis results in Figure 5c. This comparative analysis implies that the temperature during selective laser sintering with $0.42 \mathrm{~W}$ average power does reach above $800^{\circ} \mathrm{C}$ locally. Further energy accumulation and dissipation analysis, however has confirmed that the rate of heat dissipation might be of the order of $\sim 10^{6} \mathrm{Ks}^{-1}$ or so, as a consequence of which the high temperature pyrophosphate phase is preserved. Independent temperature measurements on the irradiated mineral surface using fine wire thermocouple buried below $\sim 0.5 \mathrm{~mm}$ into the synthetic mineral coating showed a cyclical variation between room temperature and $40{ }^{\circ} \mathrm{C}$ occurs, during $0.3 \mathrm{~s}$ radiation dose at each spot of $30 \mu \mathrm{m}$ diameter.



Figure 1. A computer-aided-design of combined materials and laser power delivery system.



Figure 3. Pre-programmed laser tracks for selective laser sintering synthetic minerals.

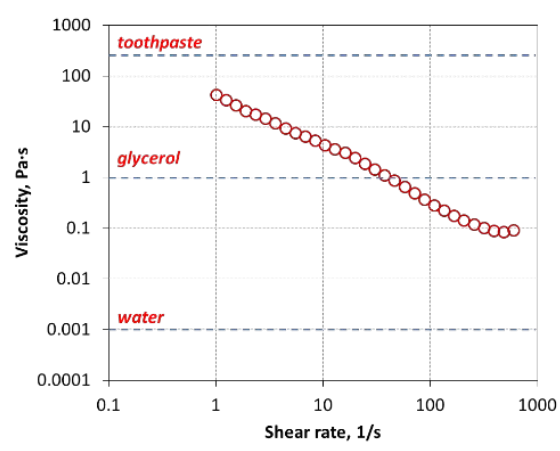

Figure 2. Viscosity of brushite mineral in ethanol.

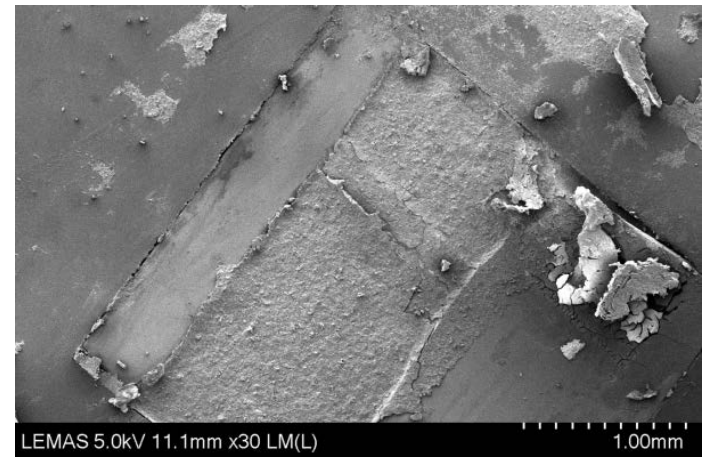

Figure 4. An SEM image of the acid lesion filled and selectively sintered with bio-minerals in the eroded bovine enamel.



Figure 5.(a)



Figure 5.(b)



Figure 5.(c)

Figure 5a shows a low magnification cross-section of enamel; $5 \mathrm{~b}$ is a high-magnification image of the microstructures showing different regions phase transformation which are mapped on the STA trace in Fig. 5c. Zone 1 is brushite/monetite, zone 2 is mixture of monetite and $\gamma$-calcium pyrophosphate and zone 3 is $\beta$-calcium pyrophosphate.

\section{ACKNOWLEDGEMENTS}

We acknowledge the support from EU LUSTRE, EU-PREFACTO and EPSRC LUMIN projects. 


\section{REFERENCES}

[1] M. Vallet-Regí and D. Arcos Navarrete: RSC Nanoscience \& Nanotechnology, no. 39, Nanoceramics in Clinical Use: From Materials to Applications, 2nd Ed., (2015), pp.1-29.

[2] M. M. Martino, P. S. Briquez, K. Maruyama, J.A. Hubbell, Advanced Drug Delivery Reviews 94 (2015) 41-52.

[3] A.D. Anastasiou, C.L. Thomson, S.A. Hussain, T.J. Edwards, S. Strafford, M. Malinowski, R. Mathieson, C. Tom, A. Brown, A.P. Brown, M.S. Duggal, A. Jha, Materials \& Design, 101 (2016) 346-354.

[4] E. Elmadani, A. Jha, T. Perali, C. Jappy, D. Walsh, C. Leburn, T. Brown, W. Sibbett, M. Duggal, J. Toumba, J. Am. Ceramic Soc. 95 (2012) 2716-2724.

[5] A.D. Anastasiou et al., Materials Science \& Engineering C, 75 (2017) 885-894. 